\title{
Enrico Berti, in memoriam
}

\author{
di Gabriele Civello
}

Un peculiare fil rouge legava la mia esistenza a quella di Enrico Berti (1935-2022): lui era allievo diretto di Marino Gentile (1906-1991); quest'ultimo era il padre di Francesco Gentile (1936-2009), il quale, infine, è stato mio maestro di filosofia del diritto nell'Università di Padova, dal primo anno del millennio fino alla sua prematura scomparsa. Il nostro legame non era, dunque, formalmente universitario, poiché un anello di questa strana catena era la "semplice" paternità di Marino Gentile - che, in realtà, fu un'autentica e piena maestria - nei confronti del figlio Francesco; tuttavia, l'aria accademica che si respirava era quella comune: quella della "Scuola padovana" di metafisica classica, filtrata attraverso la problematicità pura di Marino Gentile, e che vede nei suoi allievi Enrico Berti, Franco Chiereghin e Gianni Romano Bacchin i suoi esponenti di spicco.

I nostri incontri periodici - i cui fitti appunti conservo in alcuni quadernetti, insieme a un'intervista ancora inedita - avvenivano sempre nel suo spoglio ufficio presso lo storico "Palazzo del Capitanio", proprio in quella acciottolata Piazza Capitaniato in cui aveva vissuto Francesco Gentile, in prossimità dell'antica reggia dei Carraresi in Padova.

Il professor Berti, devo dire, osservava con curiosità i miei maldestri tentativi - da mezzo filosofo del diritto e mezzo avvocato penalista - di addentrarmi temerariamente nella filosofia estetica, in quella pratica e persino nel Sancta Sanctorum della filosofia teoretica; forse ciò che gli garbava, pur a fronte della mia preparazione filosofica poco più che liceale, era il mio tentativo di dirmi aristotelico-tomista in una giungla, quella dei giuristi contemporanei, che da almeno due secoli vede con sospetto e persino con disprezzo parole come "essenza", "ontologia" o "teleologia". 
Il nostro punto di incontro era altrettanto singolare: io provenivo, un po' impolverato, dall'irto sentiero del Tommaso d'Aquino proto-filosofo del diritto, avventurandomi e "arretrando" a grandi falcate verso i paraggi del Peripato. Berti, dalla culla un po' esoterica dei Vorsokratiker, mi accoglieva a braccia aperte nella casa di Aristotele, spaziando poi con la sua enorme cultura fino al pensiero dei nostri giorni. Ricordo ancora le bellissime conversazioni su Quando Heidegger era ancora “aristotelico”, come recita il titolo di un suo celebre saggio, dal quale traspariva il grande interesse di Berti per Sein und Zeit, molto meno per lo Heidegger post-Kehre.

Ammiravo l'estrema disinvoltura con la quale, conservando sempre un registro perfettamente piano e comprensibile, egli balzava da un misterioso frammento di Eraclito a una qualsiasi opera di Gadamer, MacIntyre, Jonas o Nussbaum, passando per un Cartesio, un Kant o uno Heidegger con quell'istinto venatorio di chi riesce a scovare persino l'Aristotele che è nascosto nei più apparenti anti-aristotelici dei filosofi moderni e contemporanei.

Il suo "aristotelismo-non-tomista" era l'impercettibile linea ideale che, per modo di dire, ci separava; ma diventavamo subito grandi alleati, nel nostro comune pluralismo ontologico, allorquando la conversazione passava nella Brescia di Via Callegari, presso la casa (oggi casa-museo) di Emanuele Severino. In quel caso, Berti mi incitava a patrocinare la nostra "fede" nell'Essere $\pi \mathrm{o} \lambda \lambda \alpha \chi \tilde{\omega} \varsigma \quad \lambda \varepsilon \gamma o ́ \mu \varepsilon v o v$, contro quell'impressionante monismo ontologico di cui Severino era uno degli ultimi strenui sostenitori. Ricordo ancora quando, in occasione di una call for paper severiniana, il prof. Berti mi inviò una bella lettera di incoraggiamento, scrivendomi:

Caro Civello, ho letto il suo abstract e mi è sembrato ottimo. Si tratta di una critica, garbata ma ferma, della posizione di Severino, o meglio delle sue conseguenze. È scritto bene, è documentato, quindi la Sua comunicazione dovrebbe essere accettata. Mi tenga al corrente sugli sviluppi; 
In effetti, venne accettata e pubblicata, anche grazie ai suggerimenti del professore.

Quando poi recensii Dike (2015) di Severino per una rivista di diritto penale, il prof. Berti mi disse che ero stato fin troppo «tenero e gentile» (sic) con l'allievo di Gustavo Bontadini, e che avrei dovuto persino criticarlo nella sua traduzione iniziale del celebre Spruch des Anaximander sulla giustizia!

Un garbo mite e cordiale era il tratto distintivo che tutti riconoscevamo da lontano nella persona di Enrico Berti; garbo però sempre asciutto, mai cerimonioso, anzi a tratti schivo e spartano, di quella semplicità di chi, essendo impegnato in pensieri alti, non bada ai convenevoli ma va subito all'essenziale.

Egli non si sottraeva mai ai miei dubbi e alle richieste di chiarimento, persino quando i quesiti venivano formulati in modo senz'altro improprio o addirittura errato; proprio qualche tempo fa, ricordo che una risposta del professore cominciò "tirandomi le orecchie", e cioè dicendo che il mio quesito era troppo ampio e problematico, che avrebbe richiesto anni di studio e di approfondimento ma che, nondimeno, egli «proprio non resisteva» - questo fu il verbo usato - alla tentazione di rispondermi ugualmente.

Ho ricevuto l'ultima lettera dal prof. Berti il 30 novembre dell'anno che è appena terminato. Gli avevo chiesto un chiarimento su Etica Nicomachea, I, 4 (1096 b 25), laddove si afferma che il "bene" non è un termine sinonimo (univoco) bensì omonimo, lasciando tuttavia aperto il quesito su quale specifico tipo di omonimia venga qui in rilievo.

Sapevo che il professore stava poco bene, ma non che fosse agli sgoccioli dei suoi giorni. Dopo nemmeno trenta minuti, egli mi avrebbe già letto e risposto:

Caro Civello, le rispondo brevemente, perché non sto bene.

Aristotele dice che il bene è omonimo, ma non indica mai un primo tra i suoi sensi. Io credo che sia omonimo per analogia di proporzionalità, quindi senza un "primo analogato". Invece Tommaso lo ammette, credo che sia Dio. 
Aristotele tratta il tema in Etica Eudemea I 8.

Nel 1979, mi pare, un certo Bärthlein ha scritto un libro per sostenere che il bene non è un omonimo "pros hen", come per Tommaso.

Cordialmente,

EB. 